\title{
Charge Carrier Mapping for Z-scheme Photocatalytic Water Splitting Sheet by Categorization of Microscopic Time-resolved Image Sequence
}

\section{Makoto Ebihara}

Chuo University

\section{Takeshi Ikeda}

TOTO Ltd.

\section{Sayuri Okunaka}

Japan Technological Research Association of Artificial Photosynthetic Chemical Process (ARPChem) Hiromasa Tokudome

Japan Technological Research Association of Artificial Photosynthetic Chemical Process (ARPChem)

\section{Kazunari Domen}

The University of Tokyo https://orcid.org/0000-0001-7995-4832

Kenji Katayama ( $\nabla$ kkata@kc.chuo-u.ac.jp )

Chuo University https://orcid.org/0000-0003-3278-6485

\section{Article}

Keywords: z scheme, water splitting, photoexcited carrier dynamics, microscopy, spectral clustering

Posted Date: March 11th, 2021

DOI: https://doi.org/10.21203/rs.3.rs-276945/v1

License: (c) (i) This work is licensed under a Creative Commons Attribution 4.0 International License. Read Full License

Version of Record: A version of this preprint was published at Nature Communications on June 17th, 2021. See the published version at https://doi.org/10.1038/s41467-021-24061-4. 


\section{Charge Carrier Mapping for Z-scheme Photocatalytic Water}

\section{Splitting Sheet by Categorization of Microscopic Time-resolved}

\section{Image Sequence}

4

5 Makoto Ebihara ${ }^{1}$ Takeshi Ikeda, ${ }^{2,3}$ Sayuri Okunaka, ${ }^{2,3}$ Hiromasa Tokudome, ${ }^{2.3}$ Kazunari

6 Domen, ${ }^{4,5}$ and Kenji Katayama ${ }^{*}$

$7 \quad{ }^{1}$ Department of Applied Chemistry, Chuo University, Tokyo 112-8551, Japan

82 Research Institute, TOTO Ltd., Kanagawa 253-8577, Japan

93 Japan Technological Research Association of Artificial Photosynthetic Chemical Process 10 (ARPChem), Tokyo 101-0032, Japan

114 Research Initiative for Supra-Materials, Interdisciplinary Cluster for Cutting Edge Research, 12 Shinshu University, Nagano 380-8553, Japan

135 Office of University Professors, The University of Tokyo, Tokyo 113-8656, Japan

*Corresponding author:

K. Katayama, Phone: +81-3-3817-1913, E-mail: kkata@kc.chuo-u.ac.jp

\section{Abstract}

Photocatalytic water splitting system using particulate semiconductor materials is a promising strategy for converting solar energy into hydrogen and oxygen. In particular, visible-light-driven 'Zschematic' printable photocatalyst sheets are cost-effective and scalable systems. However, little is known about the fundamental photophysical processes, which are key to explain and promote photoactivity. Here, we applied the pattern-illumination time-resolved phase microscopy (PI-PM) for the printed photocatalyst sheet, composed of Mo-doped $\mathrm{BiVO}_{4}$ and $\mathrm{Rh}$-doped $\mathrm{SrTiO}_{3}$, indium tin oxide (ITO) as an electron mediator, to investigate photo-generated charge carrier dynamics. Using the PIPM, we successfully observed for the first time the position- and structure-dependent charge carrier behavior, including visualization of the active/inactive sites in the sheet, under the visible-light irradiation via the time sequence images and the clustering analysis. This combination methodology could not only lead to the maximum performance of photocatalyst sheets but also applicable to other systems involving electron transfer.

Keywords: z scheme, water splitting, photoexcited carrier dynamics, microscopy, spectral clustering 


\section{Introduction}

Water splitting into hydrogen and oxygen by sunlight energy is one of the most promising clean energy resources in the future, and tremendous efforts have been made mostly for the development of new materials, ${ }^{1-3}$ and extended for the device fabrication and large-scale development. ${ }^{4,5}$ In the overall water splitting, careful selection of the band positions is necessary both for the reduction of proton and the oxidation of water, and co-catalysts are necessary to promote the reactions. New materials have emerged one after another, and one of the materials which can split water using a single semiconductor photocatalyst, aluminum-doped strontium titanate $\left(\mathrm{SrTiO}_{3}: \mathrm{Al}\right)$, showed high efficiency with an apparent quantum efficiency of almost $100 \%$ overall water splitting efficiency under UV light illumination in $2020,{ }^{6}$ and the mechanism had been studied. ${ }^{7}$ Alternatively, two different materials are used by combination; each one is used for hydrogen and oxygen generation, and the remaining charges are compensated by charge transfer between two materials, called as ' $Z$-scheme water splitting system.' $1,2,8-10$

In the Z-scheme strategy, various combinations of two different semiconductors were introduced. One of the favored combinations is $\mathrm{BiVO}_{4}$ and $\mathrm{Rh}$ doped $\mathrm{SrTiO}_{3}\left(\mathrm{SrTiO}_{3}: \mathrm{Rh}\right)$ as oxygen and hydrogen generation catalysts. In the earlier stage of the Z-scheme researches, the photo-excited electrons in $\mathrm{BiVO}_{4}$ were transferred to $\mathrm{SrTiO}_{3}: \mathrm{Rh}$ via a redox shuttle in an aqueous solution. Still, the number of candidates for redox shuttles is limited, such as $\mathrm{IO}^{3} / \mathrm{I}^{-}, \mathrm{Fe}^{2+} / \mathrm{Fe}^{3+}$. As a next step, the Z-scheme was demonstrated without using the redox mediator by direct contact between $\mathrm{BiVO}_{4}$ and $\mathrm{SrTiO}_{3}: \mathrm{Rh}$ semiconductors, ${ }^{11}$ where the photo-excited electrons and holes in each photocatalyst recombine directly at the interface. The conductive binders have been searched and introduced to promote charge transport between two materials. Alternatively, particulate photocatalyst sheets, wherein the photocatalyst particles are fixed onto a glass substrate, have been reported in recent years. For example, a physical vapor deposition (PVD)-based photocatalyst sheet composed of $\mathrm{SrTiO}_{3}: \mathrm{Rh}, \mathrm{La}$ and $\mathrm{BiVO}_{4}$ :Mo embedded in an evaporated Au layer can split water under solar light with solar-tohydrogen conversion efficiency of $1.1 \% .^{12,13}$ Also, we have recently developed a new-type photocatalyst sheet named a printable photocatalyst sheet, which can be prepared via facile and extensive screen-printing method incorporating a conductive colloidal binder (e.g., Au, ITO) with a highly-packed film structure. ${ }^{13-15}$ However, the STH was merely $0.4 \%,{ }^{15}$ and it is necessary to understand the electron transfer process and design the optimal film structure for achieving STH > $1 \%$.

In the water splitting by photocatalysts, it is essential how efficiently photo-excited charge carriers are separated and utilized for water oxidation and reduction without losing them due to recombination. So far, charge carrier dynamics in various photocatalyst particles and films, including $\mathrm{SrTiO}_{3}$ or $\mathrm{BiVO}_{4}$, have been studied. Transient absorption and time-resolved photoluminescence have 
been frequently utilized to understand the processes. Concerning $\mathrm{SrTiO}_{3}$, various doping causes trap sites, and the lifetime of the charge carriers was extended from microseconds to milliseconds, and the effect was studied in relevance to the photocatalytic activity. In several reports, doping of a single element induced a recombination center and reduced the charge carriers, but co-doping could suppress the recombination. ${ }^{16-18}$ The charge carrier dynamics was also studied for $\mathrm{BiVO}_{4}$ on the ultrafast and wide time range. The effects of trap states were discussed mostly for elongation of hole lifetimes, ${ }^{19-22}$ and the effect of active and inactive oxygen defects were clarified. ${ }^{23}$ Also, a heterojunction of $\mathrm{BiVO}_{4}$ with $\mathrm{WO}_{3}$ was studied and revealed that the junction effectively removed electrons at the interband states, ${ }^{24}$ providing a benefit for water oxidation on a millisecond to second order..$^{25}$ combination of the measurement of the refractive index change and its accompanying original analysis method called spectral clustering method, instead of the absorption change or photoluminescence..$^{26,27}$ As for the former measurement approach, the monitorization via the refractive index change has merit to favor the detection of the interfacial charge transfer because the limitation of the detection wavelength is relaxed due to the broad wavelength response. So far, this method has been applied for studying charge transfer at the interfaces of the photocatalyts ${ }^{28}$ and dye-sensitized solar cells. ${ }^{29}$ For detecting the refractive index change, we used the transient grating (TG) method and studied the dynamics of the charge carriers for $\mathrm{TiO}_{2},{ }^{30-32}$ and hematite photoanodes. ${ }^{33-35}$

The charge carrier behavior depends on the local structure and is inhomogeneous in nature for the photocatalysts, typically composed of calcinated particles and aggregates. Many researches have been devoted for studying the spatio-temporal behavior of charge carrier dynamics by using TA and photoluminescence microscopy on micro-scales, ${ }^{36-38}$ and the photocurrent behavior by microscopic photo-electrochemical measurements. ${ }^{39,40} \mathrm{We}$ also have extended our measurements of the refractive index change for a local mapping of the transient responses of photo-excited charge carriers. (patternillumination phase microscopy (PI-PM)). By illuminating a pattern of light, the sequence of images due to the refractive index change was obtained, and the image quality was recovered by the image recovery calculation techniques. ${ }^{41}$ The lifetime distribution of the charge carriers for a $\mathrm{TiO}_{2}$ particulate film was obtained, and the research clarified a broad range of the lifetime of charge carriers. ${ }^{42}$ Furthermore, the local responses of charge carriers were categorized by the spectral clustering method and found the hidden local responses of the non-radiative exciton relaxation for higher pump intensities. ${ }^{43}$ Thus, using the combination of PI-PM and the spectral clustering method, we can investigate the position- and structure-dependent charge carrier behavior under light irradiation, and also assign the carrier at specific positions.

These findings have motivated us to apply this combination of PI-PM and clustering analysis method for one of the most promising Z-scheme water splitting materials, $\mathrm{BiVO}_{4}: \mathrm{Mo} / \mathrm{SrTiO}_{3}: \mathrm{Rh}$ with a conductive colloidal binder (ITO), as a printable photocatalyst sheet with a conductive colloidal 
103 binder (ITO) prepared by the printing method. We could visualize the spatially-resolved photocatalytic 104 activity by the categorization of the charge carrier behaviors. This new methodology could detect the 105 real active and inactive sites in the photocatalytic device and will support the optimization of the active 106 structure of the photocatalyst.

107

$108 \quad$ Result and Discussion

109 The time-resolved experiments were performed on the visible-light responsible printed 110 photocatalyst sheet composed of $\mathrm{SrTiO}_{3}: \mathrm{Rh}, \mathrm{BiVO}_{4}: \mathrm{Mo}$, and ITO (STOR/ITO/BVOM) in two 111 different solvents (acetonitrile (ACN) and water). Figure 1 shows the image sequences of the refractive 112 index change for $\mathrm{SrTiO}_{3}: \mathrm{Rh} / \mathrm{ITO} / \mathrm{BiVO}_{4}: \mathrm{Mo}$ (STOR/ITO/BVOM) in different solvents ((a) in ACN, 113 (b) in water) observed by the PI-PM method. The irradiated region by the pump light corresponds to 114 the brighter regions in the images. The contrast of images originated from the refractive index change 115 due to photo-excited charge carriers in these regions. Although the images in ACN retained overall 116 brightness until 10-100 $\mu$ s, the bright regions in water gradually faded out for $1 \mu \mathrm{s}$. The overall 117 refractive index change decayed due to the charge recombination; however, images in ACN did not 118 show a simple single-component decay. Furthermore, it was noticed that the brightness in the excited regions in images strongly depended on the sample position, meaning that the materials in this sample 
(a)
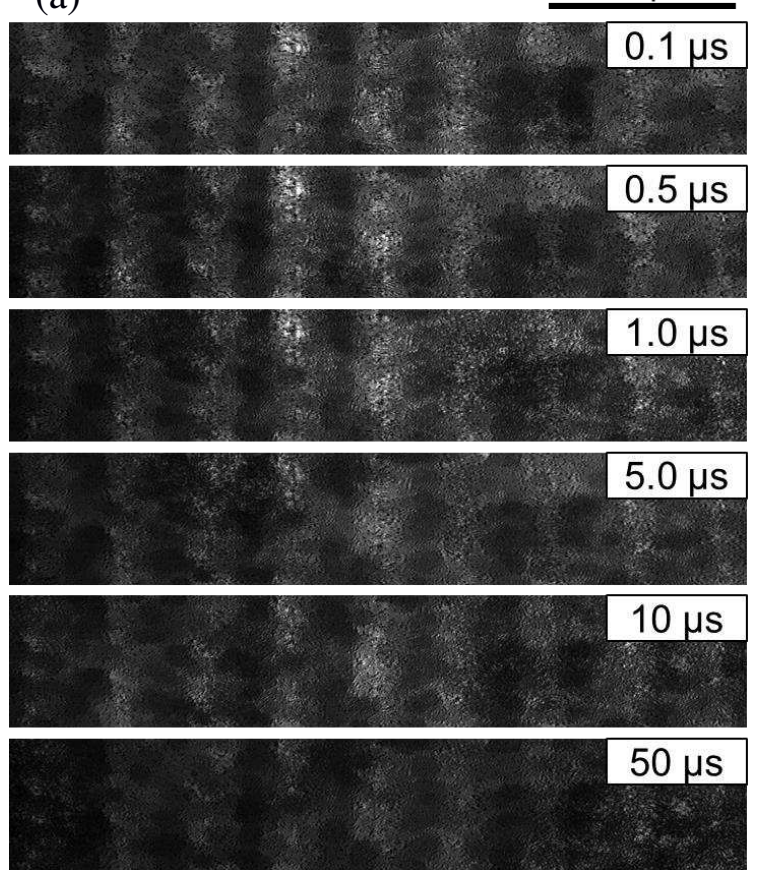

(c)
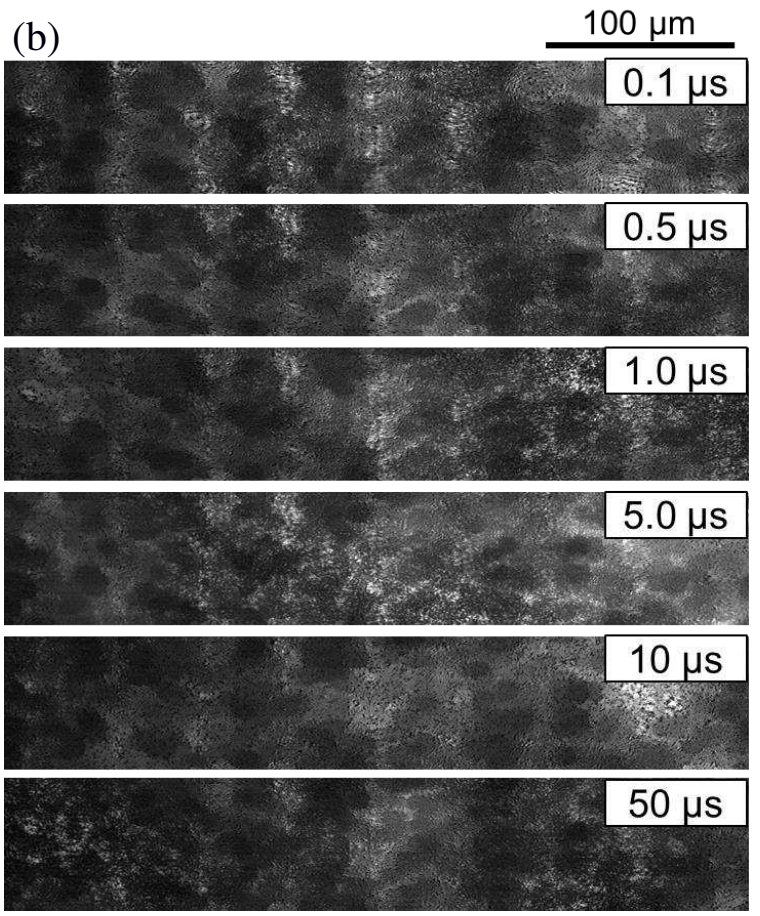

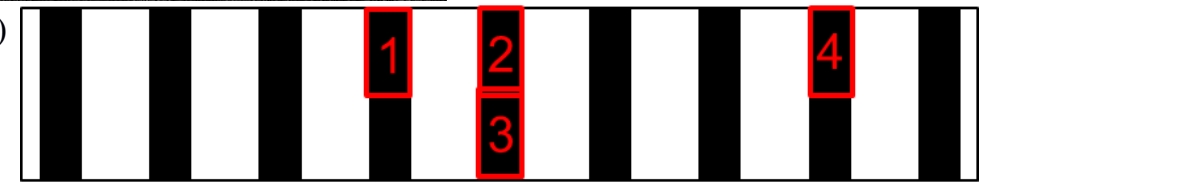

121 Fig.1 The image sequence of the refractive index change after photo-excitation by a UV pump

122 light for $\mathrm{SrTiO}_{3}: \mathrm{Rh} / \mathrm{ITO} / \mathrm{BiVO}_{4}: \mathrm{Mo}$ (STOR/ITO/BVOM) Z-scheme water splitting sheet measured by 123 the patterned-illumination time-resolved phase microscope (PI-PM) in different solvents ((a) in ACN, 124 (b) in water). (c) The light intensity pattern of the pump light and the selected regions for the cluster 125 analysis is indicated in red squares. The result of the cluster analysis for area No.1 is shown in Fig.5126 7, and the others are presented in Fig.S4-S8 in Supporting information (SI)).

For the assignment of the response on the STOR/ITO/BVOM photocatalyst sheet in the two different solutions, the temporal changes of the average contrast change of the refractive index images were obtained, which were calculated by taking the Fourier amplitude of the periodic stripe pattern at 131 the spatial frequency corresponding to the inverse of the stripe spacing, and it is shown in Fig.2(a). 132 The detailed calculation process was written in the previous papers. ${ }^{41,42}$ The signal response for the $133 \mathrm{STOR} / \mathrm{ITO} / \mathrm{BVOM}$ in ACN were categorized into three response components; rapidly increased for $13430 \mathrm{~ns}$ and decayed for $100 \mathrm{~ns}$ (first response), an additional rising for $1 \mu \mathrm{s}$ and decayed for a couple 135 of $\mu$ s (second response), a plateau until $10 \mu$ s and decay for $100 \mu$ s (third response). 
(a)

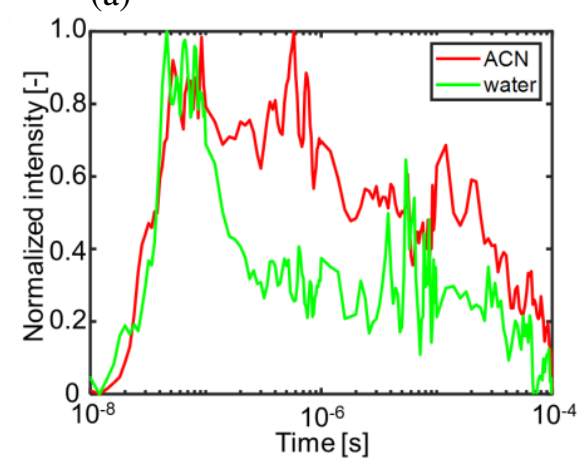

(b)

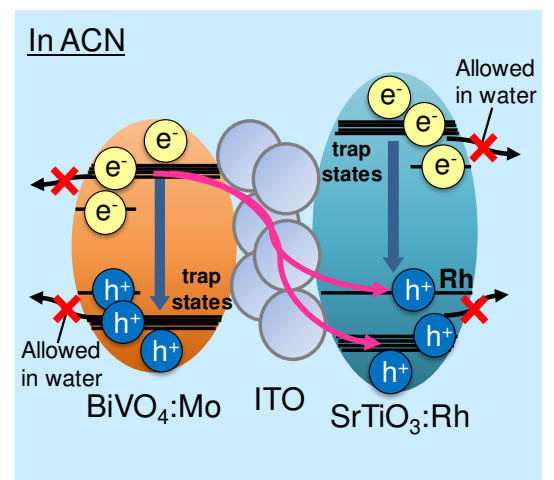

Fig.2 (a) The average refractive index change response of STOR/ITO/BVOM until $100 \mu$ s in acetonitrile (ACN) and water obtained from the PI-PM image sequence (Fig.1). The response was obtained from the signal amplitude at the light irradiated regions. (b) A schematic drawing of the charge transfer/decay processes for STOR/ITO/BVOM in ACN. Surface trapped carriers cannot be transferred to the solution side in $\mathrm{ACN}$, where all the processes of charge dynamics are completed in the system.

As shown in Fig. 2(b), photo-excited charge carriers were generated in the conduction band and valence band after the excitation because the wavelength of the pump laser was $355 \mathrm{~nm}$, and its energy exceeds the bandgap energy of both materials $\left(\mathrm{BiVO}_{4}=2.4 \mathrm{eV}, \mathrm{SrTiO}_{3}=3.2 \mathrm{eV}\right)$. Therefore, we monitored the charge carrier dynamics after the interband transition of both materials. These charge carriers were rapidly trapped to shallow or deep trap states originated from multiple types of defect. This trapping process almost finishes within the picosecond time region..$^{21,22,44,45}$ These trapped charge carriers decayed due to the recombination inside the materials or the extraction outside the materials. The first component can be considered as the mixture of these fast charge carrier dynamics. Since $\mathrm{ACN}$ is an inert solvent for charge carriers, they were confined inside the materials. Thus, these carriers remained in a slower time scale, and the response was observed until hundreds of microseconds, which corresponds to the microsecond recombination for $\mathrm{BiVO}_{4}$ and $\mathrm{SrTiO}_{3},{ }^{16,25}$ 
included in the third component.

On the other hand, the second component in ACN disappeared in the water. It indicates that the second component (100 ns to $1 \mu \mathrm{s}$ ) in ACN was attributed to electrons in STOR or holes in BVOM on the surfaces because these surface trapped charge carriers were consumed by water and utilized for water splitting reactions. The loss of the component by water indicates that the corresponding charge carriers were used for the water-splitting reactions, which are ensured by the fact that this photocatalytic sheet had a high water splitting efficiency even for pure water (Fig. S11 in SI). Vise versa, this result demonstrated that the surface trapped carriers were effectively extracted to water in this system.

In terms of the Z-scheme water splitting system, the working mechanism is complicated, and many processes of photo-excited charge carriers are included in the response. To simplify the assignment, we investigated the charge carrier dynamics of BVOM and STOR separately, using the same materials used for the Z-scheme system. Figure 3 shows the refractive index responses obtained for BVOM and STOR in ACN and water, respectively. We could confirm the second components (100 ns to $1 \mu \mathrm{s}$ ) were observed in ACN in both samples, and they disappeared in water in both cases. These results supported that these second components were attributed to the holes in BVOM and electrons in STOR, respectively, and they were utilized for water splitting reactions, as shown in Fig.2(b). It is supposed that the holes in BVOM and electrons in STOR for water splitting were observed as a mixture in the response of the Z-scheme sample because these lifetimes matched by chance. In addition to this finding, we could recognize that the second rising component was delayed until $10 \mu$ s in STOR in ACN. STOR has a mid-gap state corresponding to the $\mathrm{Rh}^{3+/ 4+}$ state within the bandgap. $\mathrm{Rh}$ is doped for the visible light absorption via the transition from the $\mathrm{Rh}^{3+}$ state to the conduction band and the valence band to the $\mathrm{Rh}^{4+}$ state, corresponding to 2.3 and $2.7 \mathrm{eV}$, respectively..$^{18,46}$ The considerable delay of the response in STOR implies that the response of STOR in $\mathrm{ACN}$ included unique components of photo-excited charge carriers due to the loss of the watersplitting reactions in $\mathrm{ACN}$ instead of water. 

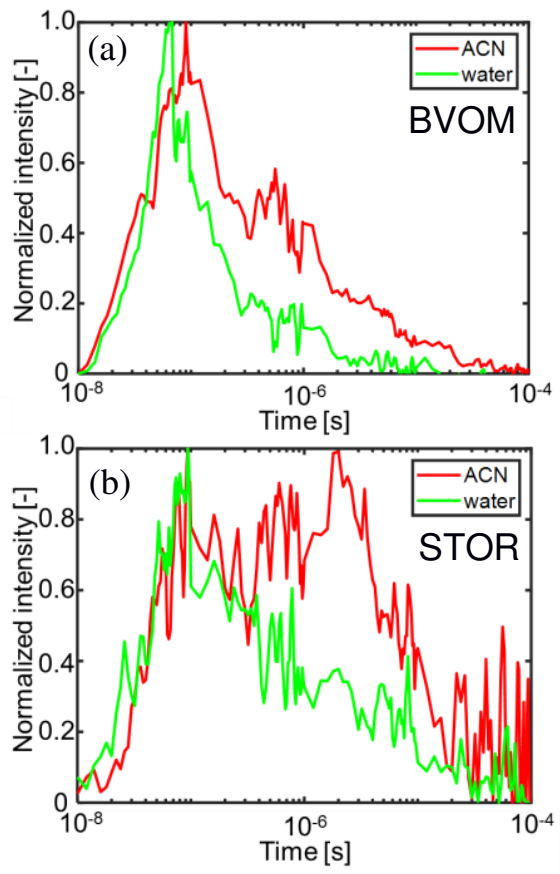

Fig.3 The average responses of the refractive index changes for (a) BVOM and (b) STOR until $100 \mu$ s in ACN and water obtained by the PI-PM image sequences.

A general understanding of the mechanism of the Z-scheme system is explained here, based on the description provided by Osterloh, et al. ${ }^{47}$ By irradiation of the visible light, both BVOM and STOR are photo-excited, and the photo-generated holes in BVOM and photo-generated electrons in STOR are used for the water splitting to oxygen and hydrogen, respectively. Photo-excited electrons in BVOM are transferred to the neighboring STOR particles, and the $\mathrm{Rh}^{4+}$ elements are reduced to $\mathrm{Rh}^{3+}$ by the electron transfer. The $\mathrm{Rh}^{4+}$ elements work as the electron acceptor in this case. With regard to STOR, electrons are excited from the $\mathrm{Rh}^{3+}$ state to the conduction band for the visible light absorption, causing $\mathrm{Rh}^{4+}$ species generation. Water splitting continues when these reactions and charge transfer cycles repeatedly occur. It was reported that the $\mathrm{Rh}^{4+}$ states reduce the activity of water splitting by working as the recombination sites for the electrons in the conduction band, and much effort has been made to reduce the $\mathrm{Rh}^{4+}$ states. ${ }^{18,46}$ In our experiments, it was confirmed that the slow rising until 10 $\mu$ s in STOR in ACN was due to the formation of the $\mathrm{Rh}^{4+}$ state by the hole transfer from the valence band to the $\mathrm{Rh}^{3+}$ states, as understood from the experiment of the methanol $(\mathrm{MeOH})$ scavenger effect, as described in detail in Fig. S1 in Supporting Information (SI).

The schematic flow of the whole charge dynamics of STOR/ITO/BVOM is summarized in Fig.4. Based on the series of results, we concluded the response included at least three components. As a first step, photo-excited charge carriers decayed for $<100 \mathrm{~ns}$ due to the intrinsic recombination processes because the responses in this time region were not influenced by water. During these processes, free charge carriers and trapped charge carriers at shallow trap states recombined inside each material. 
(first component) The processes could include the recombination between the electrons in BVOM and the holes in STOR as well because of the smooth charge transfer via the ITO binder. In the second component, until $\sim 1 \mu \mathrm{s}$, the response decayed due to the recombination of the surface-trapped charge carriers in ACN. The components were lost for water splitting when the photocatalytic sheets had contact with water. This response includes the contributions from both of the electrons in STOR and the holes in BVOM, considering the responses of STOR and BVOM only (Fig.3). The third component until $\sim 100 \mu$ s includes the hole accumulation decayed process from the valence band to $\mathrm{Rh}^{3+}$ state in STOR $(\sim 10 \mu \mathrm{s})$ because this component was observed for STOR only and was quenched well by $\mathrm{MeOH}$. The third component also includes other slower recombination processes. The hole accumulation in STOR contributed less in the Z-scheme system because ITO worked to mediate the electron transfer in BVOM to STOR and effectively reduce the $\mathrm{Rh}^{4+}$ state. However, we could confirm the accumulation of $\mathrm{Rh}^{4+}$ (slow rising component) was enhanced due to inefficient electron transfer from BVOM without the charge transfer mediator (ITO binder) (Refer to Section of 'The effect of ITO binder' and Fig.S2 and S3, for the detailed discussion on the ITO binder). In terms of the water splitting, the effective charges on each material in the second process are essential, and the high efficiency of water splitting of this Z-scheme system is possibly due to the well-matching of the lifetimes of the surface-trapped electrons in STOR and holes in BVOM. Furthermore, the oxidized $\mathrm{Rh}^{4+}$ state was reduced effectively by the smooth electron transfer from BVOM; otherwise, the state is well-known to deteriorate the hydrogen-generating electrons in STOR due to the recombination.

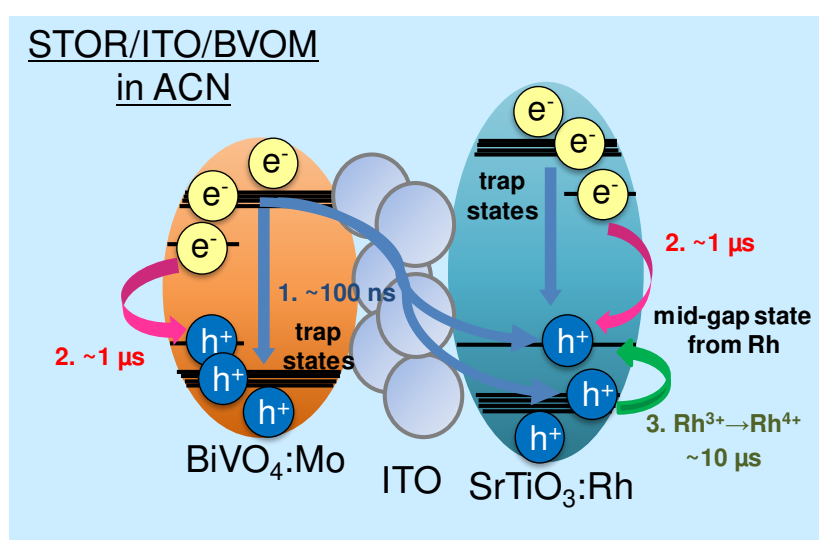

Fig.4 The schematic flow diagram of the whole charge dynamics in STOR/ITO/BVOM in ACN. Step 1 includes intrinsic recombination inside materials and charge transfer between two materials. Step 2 indicates the decay of surface trapped charge carriers in ACN, which are utilized for water splitting reactions with water outside. Step 3 includes the hole trapping to $\mathrm{Rh}^{3+/ 4+}$ state in STOR and the slower recombination. 
consisted of more than one material. Furthermore, we noticed the inhomogeneity of the responses in the image sequences measured by the PI-PM method. Hence, we categorized the position-dependent (local) responses based on their similarity by the clustering analysis of the transient image sequence. A detailed procedure of this analysis was introduced in our previous paper. ${ }^{43}$ Briefly, one of the local regions in the excited area was selected for the analysis, and the local responses in the selected region were extracted from each pixel in the image sequence. These responses were classified into several categories of the charge carrier responses based on the similarity calculated by the rise and decay profile. We applied this analysis to the local responses in the image sequence of STOR/ITO/BVOM in ACN (Fig.5). The selected position for the analysis is indicated in the red square in Fig.1(c) (No.1). Figure 5(a) shows a sequence of the temporal images of the refractive index change, representing a position-dependent response, where some micron-sized particles showed a large refractive index change with higher brightness, lasting until $1 \mu \mathrm{s}$, and fading out for $10 \mu \mathrm{s}$.
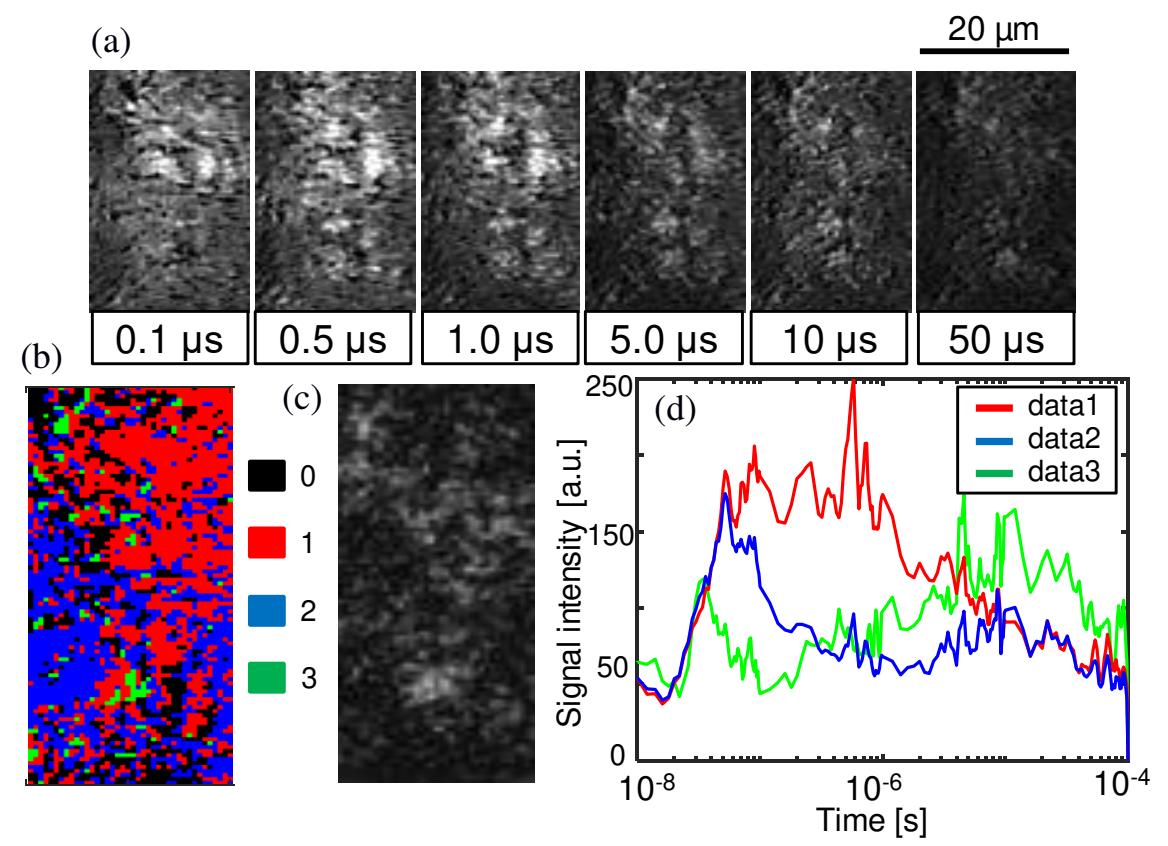

(a) An image sequence of the refractive index change for STOR/ITO/BVOM in ACN in a square region $(20 \times 50 \mu \mathrm{m})$ corresponding to No.1 in Fig.1(c) on the order from nanoseconds to microseconds. The scale bar corresponds to $20 \mu \mathrm{m}$. (b) The categorized mapping of the charge carrier responses of (a). An outlier positioned far from all categories were colored in black (\#0). (c) A microscopic image in the same area as (a). (d) The averaged responses for each category in (b) are shown.

From the spectral clustering, ${ }^{48}$ the transient responses were classified into three categories, and they were mapped out in Fig.5(b). The outliers, far from the three categories, were indicated as \#0 
(black) in this mapping. The original microscopic phase image of the sample is shown in Fig. 5(c). In the sample image, aggregations with a few microns in diameter were recognized. The bright positions in the transient image sequence were almost overlapped with the positions of the aggregations. In each category, the responses at all the pixels in the same category were averaged and shown in Fig.5(d). From Fig.5(b) and (d), the categorized map was mostly composed of red and blue regions. Based on the assignment in Fig.2(a), the response in the red region was similar to an averaged overall response (data1 in (d)), and the blue response seems to be composed of the first and third responses (data2 in (d)). There was a small portion of green regions in Fig.5(b), and it showed a slow rising component corresponding to the third component (data3 in (d)). Based on the assignment described earlier, the response of data1 in Fig.5(d) was composed of the intrinsic recombination and the decay of the surface trapped carriers until $1 \mu \mathrm{s}$ utilized for water splitting reactions. Therefore, water splitting reactions should mostly occur in the red region. The blue response was composed of the intrinsic recombination with the hole decay to $\mathrm{Rh}^{3+}$ state $\left(\mathrm{Rh}^{4+}\right.$ formation) in STOR. The area of the green regions, corresponding to $\mathrm{Rh}^{4+}$ formation in Fig.5(d), was much smaller compared with the red and blue regions. It means that the blue and green regions do not have a water-splitting activity. This analysis was applied to other regions (No.2 No.4 in Fig.1(c)), and the results are shown in Fig.S4-S6 in SI. Figure S4 and S5 had almost the same categories of the responses as Fig.5. However, the red regions mostly occupied the whole area compared with Fig.5. It means that regions No.2 and No.3 were more active for water splitting reactions than that for region No.1. On the other hand, as shown in Fig.S6, t the green region increased for region No.4. It indicated the $\mathrm{Rh}^{4+}$ formation process was observed in a large portion of this region, leading to ineffective water-splitting. Based on these results, each material was not dispersed homogeneously and mixed well on the substrate, indicating that some regions were not an ideal STOR/ITO/BVOM composition.

The same analysis was conducted in the case of a Z-scheme sample without the ITO binder (STOR/BVOM) to confirm the effect of ITO (Fig.6). As you can see from these results, the same types of categories for transient responses were found. However, the area of the green regions in the categorized map (Fig.6(b)) drastically increased, while the number of the red region decreased considerably compared with Fig.5(b). The ratio of the blue region remained almost the same. This result indicated that the area of the $\mathrm{Rh}^{4+}$ formation drastically increased if there was no ITO binder in the system. This is exactly the reason why the water splitting reaction was inefficient for this system. Additionally, the green region was observed at the positions of aggregations in the sample image (Fig.5(c)). It implies that the recognized aggregation in the sample image corresponded to the positions of the STOR particle aggregates. The same experiments and analyses were applied to other regions (No.2 and No.3 in Fig.1 (c)), and the results are shown in Fig.S7 and S8. We could confirm that the $\mathrm{Rh}^{4+}$ formation occurred in a large portion of these regions. From the combination of the transient 
water-splitting reactions proceeded and clarify the role of ITO for higher efficiency. Since the positions for the high and low efficiencies of water splitting reactions were spatially separated, it indicates that the charge transfer between BVOM and STOR were efficient in some regions and not

292 in other regions, and possibly can be improved by the mixing and deposition conditions on the 293 substrate.

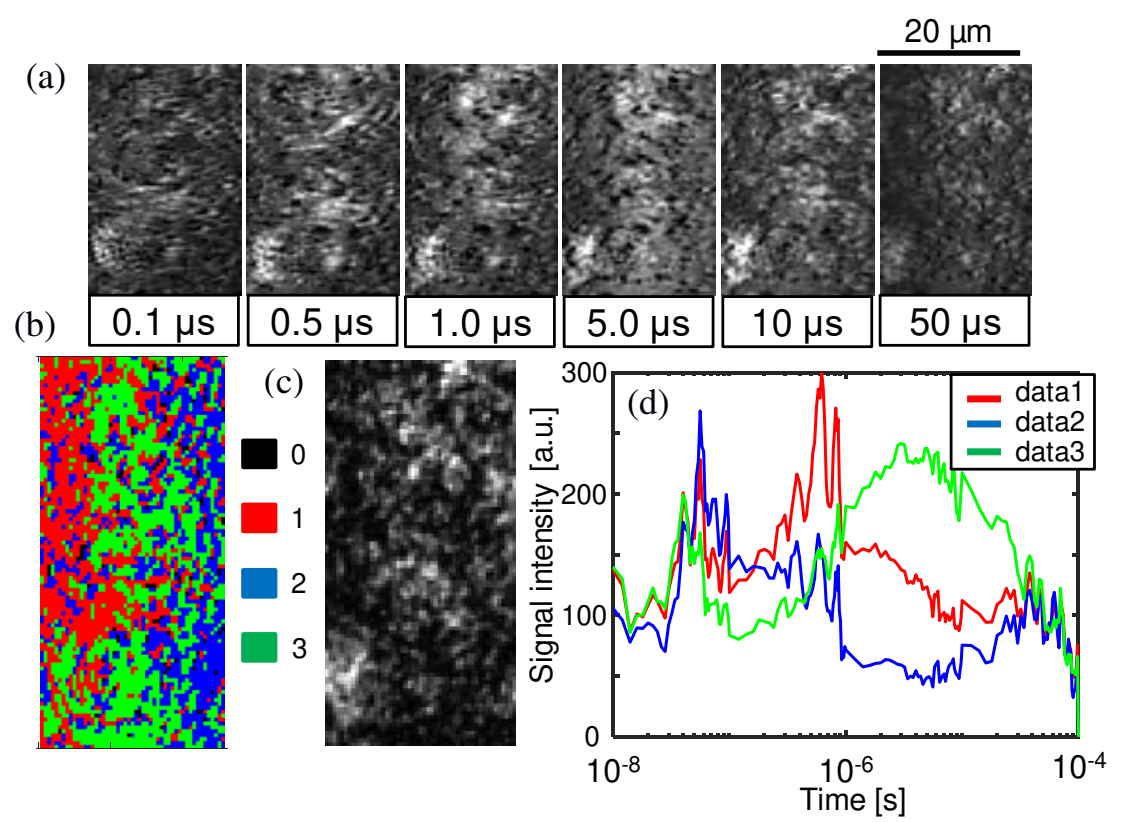

Fig.6 (a) An image sequence of the refractive index response for STOR/BVOM in ACN in a square region $(18 \times 50 \mu \mathrm{m})$ corresponding to No.1 in Fig.1(c) on the order from nanoseconds to microseconds. The scale bar corresponds to $20 \mu \mathrm{m}$. (b) The categorized mapping of the charge carrier responses of (a). An outlier positioned far from all categories were colored in black (\#0). (c) A microscopic image in the same area as (a). (d) The averaged responses for each category in (b) are shown.

Finally, we studied that the local responses of STOR/ITO/BVOM in water by using the same cluster analysis to compare the difference from the results in ACN, as shown in Fig.7. The analysis was applied for the exactly same region as the region in Fig.5, and only the solvent was replaced with water. From Fig.7 (b) and (d), the categorized map was composed of the blue and green regions, corresponding to the fast decay (data1) and the weak response (data2) regions, and we could not observe the red and green regions found under the ACN condition (Fig.5). This result indicated that all the surface trapped charge carriers in the case of ACN were utilized for water splitting reactions in water. These components were completely removed by water, and only intrinsic recombination was observed. The comparison of the results in Fig.5 and 7 ensures the reaction of water splitting on the surface. 
The categorization of local charge carrier responses with a combination of the PI-PM measurements and the clustering analyses reported herein provides useful information for designing and fabricating highly-efficient photocatalytic films or devices that have heterojunctions between different semiconductor particles. This method can positively affect a broad range of physicochemical processes that involve solid-state electron transfer.
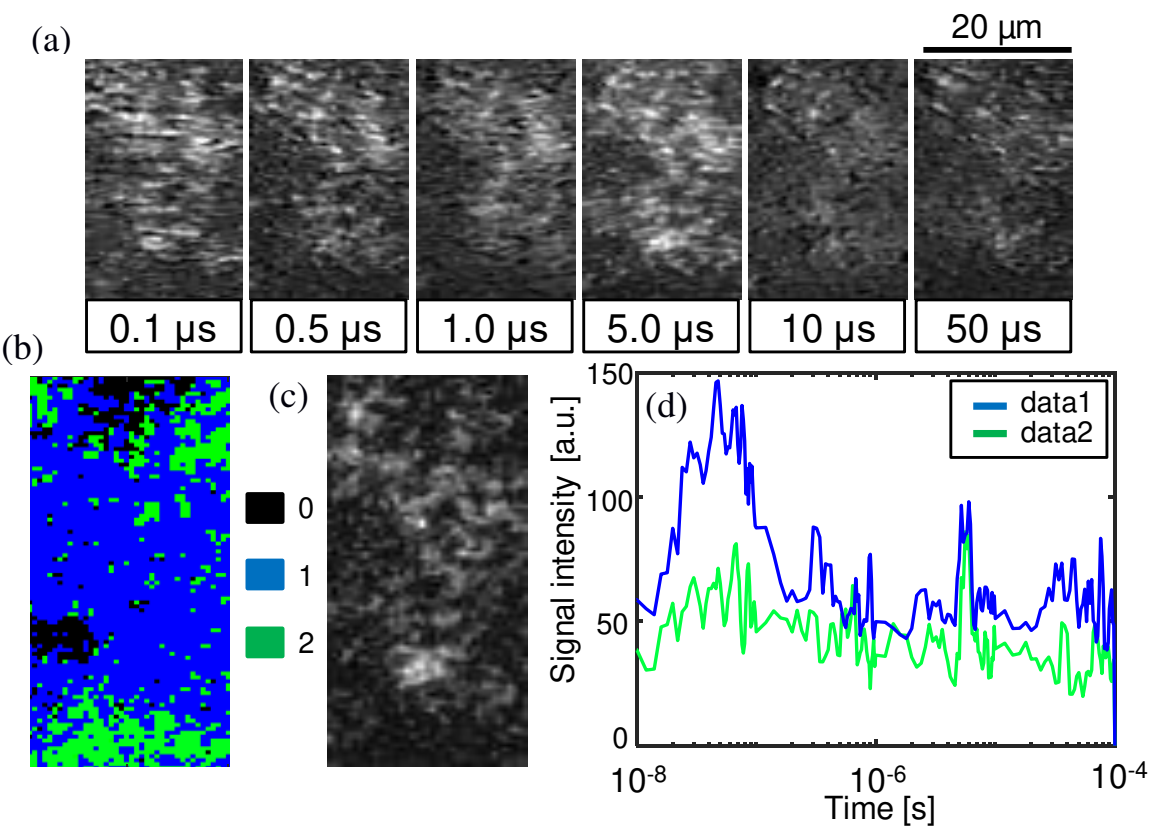

(a) An image sequence of the refractive index response for STOR/ITO/BVOM in water in a square region $(20 \times 50 \mu \mathrm{m})$ corresponding to No.1 on the order from nanoseconds to microseconds. The location of the sample was exactly the same as Fig.5(a). The scale bar corresponds to $20 \mu \mathrm{m}$. (b) The categorized mapping of the charge carrier responses of (a). An outlier positioned far from all categories was colored in black (\#0) (c) A microscopic image in the same area as (a). (d) The averaged responses for each category in (b) are shown.

\section{Conclusion}

This work revealed the origin of the efficient water splitting reactions in STOR/ITO/BVOM photocatalyst sheets that can split water to $\mathrm{H}_{2}$ and $\mathrm{O}_{2}$ under visible light by employing our original clustering analysis of photo-generated charge carrier responses in the transient image sequence. The combination of the observation technique (PI-PM) and the clustering analysis could lead to visualize reactive sites for efficient water-splitting reactions and inactive sites deteriorating water splitting 
efficiency. From the averaged responses of the refractive index change for different combinations of materials in various solvents, we could find three components, corresponding to the intrinsic recombination of photo-excited charge carriers inside materials ( 100 ns), recombination of surface trapped charge carriers which can essentially contribute to split water $(\sim 1 \mu \mathrm{s})$, and the hole trapping (accumulation) to the $\mathrm{Rh}$ state in STOR, generating $\mathrm{Rh}^{4+}$ state $(\sim 10 \mu \mathrm{s})$. Consequently, we could visually demonstrate that the region of the hole trapping to the $\mathrm{Rh}$ state, where is the region to suppress the water splitting reactions in the photocatalyst sheet, decreased in the sheet including ITO mediators at the reaction sites due to the efficient inter-particular charge transfer. This technique will be a powerful tool for the detection of active/inactive sites for the water-splitting materials but also the other materials involving electron transfer reactions, providing swift optimization for photocatalytic materials.

\section{Method}

The principle of the PI-PM is described in the previous papers. ${ }^{41,43}$ In brief, an arbitrary light pattern is irradiated on a sample to excite charge carriers. The charge carriers decay or diffuse as time passes due to the recombination, charge trapping, and transport, and the charge carrier distribution is varied in time. The distribution of the photo-excited charge carriers is observed from the refractive index change by the phase-sensitive imaging. The refractive index was imaged by the Talbot selfimaging technique. ${ }^{49}$ The pattern-illumination is required for applying various image recovery algorithms, as described in a different paper. ${ }^{41}$ In this study, only the background correction was processed before the analyses to prevent the loss of small features in images. In this optical configuration, the TA responses could also be included in the image, but the refractive index change was the major contribution to the signal, which could be confirmed by optimizing the focus position because the TA signal intensity does not depend on the focus position.

The experimental setup is fully described in Fig. S9 in SI. Briefly, the wavelengths of the pump and probe pulse lights were 355 and $532 \mathrm{~nm}$, respectively, with pulse widths of $5 \mathrm{~ns}$. The pump light was illuminated as a Ronchi ruling pattern similar to the transient grating (TG) technique, as we reported previously to understand the refractive index change response easily as the amplitude change by Fourier transform. The width of each line in the ruling-pattern was $25 \mu \mathrm{m}$, and the spacing was 45 $\mu \mathrm{m}$.

The sample was $\mathrm{Rh}$-doped $\mathrm{SrTiO}_{3}$ and Mo-doped $\mathrm{BiVO}_{4}$, including ITO nanoparticles as a conductive binder, and printed on a glass substrate with a thickness of $1 \mu \mathrm{m}$. The detailed preparation method is described in SI. The SEM images of Rh-doped $\mathrm{SrTiO}_{3}$ and Mo-doped $\mathrm{BiVO}_{4}$ are shown in Fig. S10 in SI. The average diameters of them were $300 \mathrm{~nm}$ and $2 \mu \mathrm{m}$, respectively. The gas evolution data for this photocatalyst sheet in pure water is shown in Fig. S11 in SI, and the STH was $0.4 \%$. 

rubber spacer (thickness: $0.5 \mathrm{~mm}$ ), and liquids were sandwiched within the gap. Each film sample was measured in contact with acetonitrile (ACN) and water. $\mathrm{ACN}$ is an inert solvent for photocatalytic reactions, where no charge transfer at the interface is guaranteed, ${ }^{28}$ while it occurs for water. We used

\section{Author Contribution Statement}

ME, HT and KK designed the experiments and made measurements. TI, SO, HT, and KD prepared the photocatalytic sheet. ME analyzed the data, and ME, HT, KD, and KK considered the result. ME and KK wrote the manuscript, and all the authors reviewed it.

\section{Declaration of competing interests}

I declare that the authors have no competing interests or other interests that might be perceived to influence the results and/or discussion reported in this article.

\section{Acknowledgments}

The research was financially supported by JST PRESTO (\#JPMJPR1675), and the Institute of Science and Engineering, Chuo University. 


\section{$401 \quad$ References}

402 1. Wang, Z., Li, C. \& Domen, K. Recent developments in heterogeneous photocatalysts for solardriven overall water splitting. Chem. Soc. Rev. 48, 2109-2125 (2019).

2. Wang, Q. \& Domen, K. Particulate Photocatalysts for Light-Driven Water Splitting: Mechanisms, Challenges, and Design Strategies. Chem. Rev. 120, 919-985 (2020).

3. Djurišić, A. B., He, Y. \& Ng, A. M. C. Visible-light photocatalysts: Prospects and challenges.

4. Hisatomi, T. \& Domen, K. Reaction systems for solar hydrogen production via water splitting with particulate semiconductor photocatalysts. Nat. Catal. 2, 387-399 (2019).

5. Goto, Y. et al. A Particulate Photocatalyst Water-Splitting Panel for Large-Scale Solar Hydrogen Generation. Joule 2, 509-520 (2018).

6. Takata, T. et al. Photocatalytic water splitting with a quantum efficiency of almost unity. Nature

7. Zhao, Z. et al. Electronic structure basis for enhanced overall water splitting photocatalysis with aluminum doped SrTiO3 in natural sunlight. Energy Environ. Sci. 12, 1385-1395 (2019). 

of two different $\mathrm{TiO} 2$ photocatalysts (anatase, rutile) and a IO3-/I- shuttle redox mediator. Chem. Phys. Lett. 344, 339-344 (2001).

10. Ng, B.-J. et al. Z-Scheme Photocatalytic Systems for Solar Water Splitting. Adv. Sci. 7, 1903171 (2020).

11. Full Text PDF.

12. Wang, Q. et al. Z-scheme water splitting using particulate semiconductors immobilized onto

13. Wang, Q. et al. Scalable water splitting on particulate photocatalyst sheets with a solar-to-

14. Wang, Q. et al. Printable Photocatalyst Sheets Incorporating a Transparent Conductive Mediator

15. Okunaka, S. et al. Z-Scheme Water Splitting under Near-Ambient Pressure using a Zirconium Oxide Coating on Printable Photocatalyst Sheets. ChemSusChem 13, 4906-4910 (2020).

16. Yamakata, A., Kawaguchi, M., Murachi, R., Okawa, M. \& Kamiya, I. Dynamics of 
(2020).

438

18. Furuhashi, K., Jia, Q., Kudo, A. \& Onishi, H. Time-Resolved Infrared Absorption Study of SrTiO3 Photocatalysts Codoped with Rhodium and Antimony. J. Phys. Chem. C 117, 1910119106 (2013)

19. Cooper, J. K. et al. Physical Origins of the Transient Absorption Spectra and Dynamics in ThinFilm Semiconductors: The Case of BiVO4. J. Phys. Chem. C 122, 20642-20652 (2018).

20. Aiga, N. et al. Electron-Phonon Coupling Dynamics at Oxygen Evolution Sites of Visible-LightDriven Photocatalyst: Bismuth Vanadate. J. Phys. Chem. C 117, 9881-9886 (2013).

21. Ravensbergen, J. et al. Unraveling the Carrier Dynamics of BiVO4: A Femtosecond to Microsecond Transient Absorption Study. J. Phys. Chem. C 118, 27793-27800 (2014).

22. Suzuki, Y. et al. Rational Interpretation of Correlated Kinetics of Mobile and Trapped Charge Carriers: Analysis of Ultrafast Carrier Dynamics in BiVO4. J. Phys. Chem. C 121, 19044-19052 (2017).

23. Selim, S. et al. Impact of Oxygen Vacancy Occupancy on Charge Carrier Dynamics in BiVO4 Photoanodes. J. Am. Chem. Soc. 141, 18791-18798 (2019).

24. Grigioni, I. et al. In Operando Photoelectrochemical Femtosecond Transient Absorption Spectroscopy of WO3/BiVO4 Heterojunctions. ACS Energy Lett. 4, 2213-2219 (2019).

25. Selim, S. et al. WO3/BiVO4: impact of charge separation at the timescale of water oxidation. 
Chem. Sci. 10, 2643-2652 (2019).

456

26. Katayama, K., Yamaguchi, M. \& Sawada, T. Lens-free heterodyne detection for transient grating experiments. Appl. Phys. Lett. 82, 2775-2777 (2003).

27. Okuda, M. \& Katayama, K. Selective detection of real and imaginary parts of refractive index change in solutions induced by photoexcitation using near-field heterodyne transient grating method. Chem. Phys. Lett. 443, 158-162 (2007).

28. Kuwahara, S. \& Katayama, K. Distinction of electron pathways at titanium oxide/liquid interfaces in photocatalytic processes and co-catalyst effects. Phys. Chem. Chem. Phys. 18, 25271-25276 (2016).

29. Kuwahara, S. et al. Detection of non-absorbing charge dynamics via refractive index change in dye-sensitized solar cells. Phys. Chem. Chem. Phys. 15, 5975-5981 (2013).

30. Shen, Q. et al. Photoexcited hole dynamics in $\mathrm{TiO} 2$ nanocrystalline films characterized using a lens-free heterodyne detection transient grating technique. Chem. Phys. Lett. 419, 464-468 (2006).

31. Hata, H., Katayama, K., Shen, Q. \& Toyoda, T. Characterization of Photoexcited Carriers and Thermal Properties of Nanoparticulate TiO2 Film Using Heterodyne Transient Grating Method. Jpn. J. Appl. Phys. 51, 42601 (2012).

32. Shingai, D., Ide, Y., Sohn, W. Y. \& Katayama, K. Photoexcited charge carrier dynamics of interconnected $\mathrm{TiO} 2$ nanoparticles: evidence of enhancement of charge separation at anatase- 
rutile particle interfaces. Phys. Chem. Chem. Phys. 20, 3484-3489 (2018).

474

33. Inaba, M., Katayama, K. \& Sohn, W. Y. Cooperative effects of surface and interface treatments in a hematite $(\alpha-\mathrm{Fe} 2 \mathrm{O} 3)$ photo-anode on its photo-electrochemical performance. Sustain. Energy Fuels 4, 2174-2183 (2020).

34. Sohn, W. Y. et al. Uncovering Photo-Excited Charge Carrier Dynamics in Hematite ( $\alpha$-Fe2O3) Hidden in the Nanosecond Range by the Heterodyne Transient Grating Technique Combined with the Randomly Interleaved Pulse-Train Method. J. Phys. Chem. C 123, 6693-6700 (2019).

35. Sohn, W. Y. et al. Charge carrier kinetics in hematite with $\mathrm{NiFeOx}$ coating in aqueous solutions: Dependence on bias voltage. J. Photochem. Photobiol. Chem. 353, 344-348 (2018).

36. Zhu, Y. \& Cheng, J.-X. Transient absorption microscopy: Technological innovations and applications in materials science and life science. J. Chem. Phys. 152, 020901 (2020).

37. Simpson, M. J., Doughty, B., Yang, B., Xiao, K. \& Ma, Y.-Z. Imaging Electronic Trap States in Perovskite Thin Films with Combined Fluorescence and Femtosecond Transient Absorption Microscopy. J. Phys. Chem. Lett. 7, 1725-1731 (2016).

38. Gao, Y., Nie, W., Wang, X., Fan, F. \& Li, C. Advanced space- and time-resolved techniques for photocatalyst studies. Chem. Commun. 56, 1007-1021 (2020).

39. Wang, L., Tahir, M., Chen, H. \& Sambur, J. B. Probing Charge Carrier Transport and Recombination Pathways in Monolayer MoS2/WS2 Heterojunction Photoelectrodes. Nano Lett. 

19, 9084-9094 (2019).

492

40. Todt, M. A., Isenberg, A. E., Nanayakkara, S. U., Miller, E. M. \& Sambur, J. B. Single-Nanoflake Photo-Electrochemistry Reveals Champion and Spectator Flakes in Exfoliated MoSe2 Films. $J$. Phys. Chem. C 122, 6539-6545 (2018).

41. Katayama, K. Photo-excited charge carrier imaging by time-resolved pattern illumination phase microscopy. J. Chem. Phys. 153, 054201 (2020).

42. Ebihara, M., Sohn, W. Y. \& Katayama, K. Lifetime mapping of photo-excited charge carriers by the transient grating imaging technique for nano-particulate semiconductor films. Rev. Sci. Instrum. 90, 073905 (2019).

43. Ebihara, M. \& Katayama, K. Anomalous Charge Carrier Decay Spotted by Clustering of a TimeResolved Microscopic Phase Image Sequence. J. Phys. Chem. C 124, 23551-23557 (2020).

44. Kato, K., Jiang, J., Sakata, Y. \& Yamakata, A. Effect of Na-Doping on Electron Decay Kinetics in SrTiO3 Photocatalyst. ChemCatChem 11, 6349-6354 (2019).

45. Kato, K. \& Yamakata, A. Defect-Induced Acceleration and Deceleration of Photocarrier Recombination in SrTiO3 Powders. J. Phys. Chem. C 124, 11057-11063 (2020).

46. Konta, R., Ishii, T., Kato, H. \& Kudo, A. Photocatalytic Activities of Noble Metal Ion Doped SrTiO3 under Visible Light Irradiation. J. Phys. Chem. B 108, 8992-8995 (2004).

47. Han, R., Melo, M. A., Zhao, Z., Wu, Z. \& Osterloh, F. E. Light Intensity Dependence of 
Fig.1 The image sequence of the refractive index change after photo-excitation by a UV pump light for $\mathrm{SrTiO}_{3}: \mathrm{Rh} / \mathrm{ITO} / \mathrm{BiVO}_{4}: \mathrm{Mo}$ (STOR/ITO/BVOM) Z-scheme water splitting sheet measured by the patterned-illumination time-resolved phase microscope (PI-PM) in different solvents ((a) in ACN, (b) in water). (c) The light intensity pattern of the pump light and the selected regions for the cluster analysis is indicated in red squares. The result of the cluster analysis for area No.1 is shown in Fig.57, and the others are presented in Fig.S4-S8 in Supporting information (SI)).

(a) The average refractive index change response of STOR/ITO/BVOM until $100 \mu \mathrm{s}$ in acetonitrile (ACN) and water obtained from the PI-PM image sequence (Fig.1). The response was obtained from the signal amplitude at the light irradiated regions. (b) A schematic drawing of the charge transfer/decay processes for STOR/ITO/BVOM in ACN. Surface trapped carriers cannot be transferred to the solution side in ACN, where all the processes of charge dynamics are completed in the system.

Fig.3 The average responses of the refractive index changes for (a) BVOM and (b) STOR until $100 \mu \mathrm{s}$ in ACN and water obtained by the PI-PM image sequences.

Fig.4 The schematic flow diagram of the whole charge dynamics in STOR/ITO/BVOM in ACN. Step 1 includes intrinsic recombination inside materials and charge transfer between two materials. Step 2 indicates the decay of surface trapped charge carriers in ACN, which are utilized for water splitting reactions with water outside. Step 3 includes the hole trapping to $\mathrm{Rh}^{3+/ 4+}$ state in STOR and

(a) An image sequence of the refractive index change for STOR/ITO/BVOM in ACN in a square region $(20 \times 50 \mu \mathrm{m})$ corresponding to No.1 in Fig.1(c) on the order from nanoseconds to microseconds. The scale bar corresponds to $20 \mu \mathrm{m}$. (b) The categorized mapping of the charge carrier responses of (a). An outlier positioned far from all categories were colored in black (\#0). (c) A microscopic image in the same area as (a). (d) The averaged responses for each category in (b) are shown.

(a) An image sequence of the refractive index response for STOR/BVOM in ACN in a square region $(18 \times 50 \mu \mathrm{m})$ corresponding to No.1 in Fig.1(c) on the order from nanoseconds to microseconds. The scale bar corresponds to $20 \mu \mathrm{m}$. (b) The categorized mapping of the charge carrier 
551 microscopic image in the same area as (a). (d) The averaged responses for each category in (b) are 552 shown.

553

554 Fig.7 (a) An image sequence of the refractive index response for STOR/ITO/BVOM in water in 555 a square region $(20 \times 50 \mu \mathrm{m})$ corresponding to No.1 on the order from nanoseconds to microseconds. 556 The location of the sample was exactly the same as the Fig.5(a). The scale bar corresponds to $20 \mu \mathrm{m}$. 557 (b) The categorized mapping of the charge carrier responses of (a). An outlier positioned far from all 558 categories were colored in black (\#0) (c) A microscopic image in the same area as (a). (d) The 559 averaged responses for each category in (b) are shown.

560 


\section{Figures}

(a)
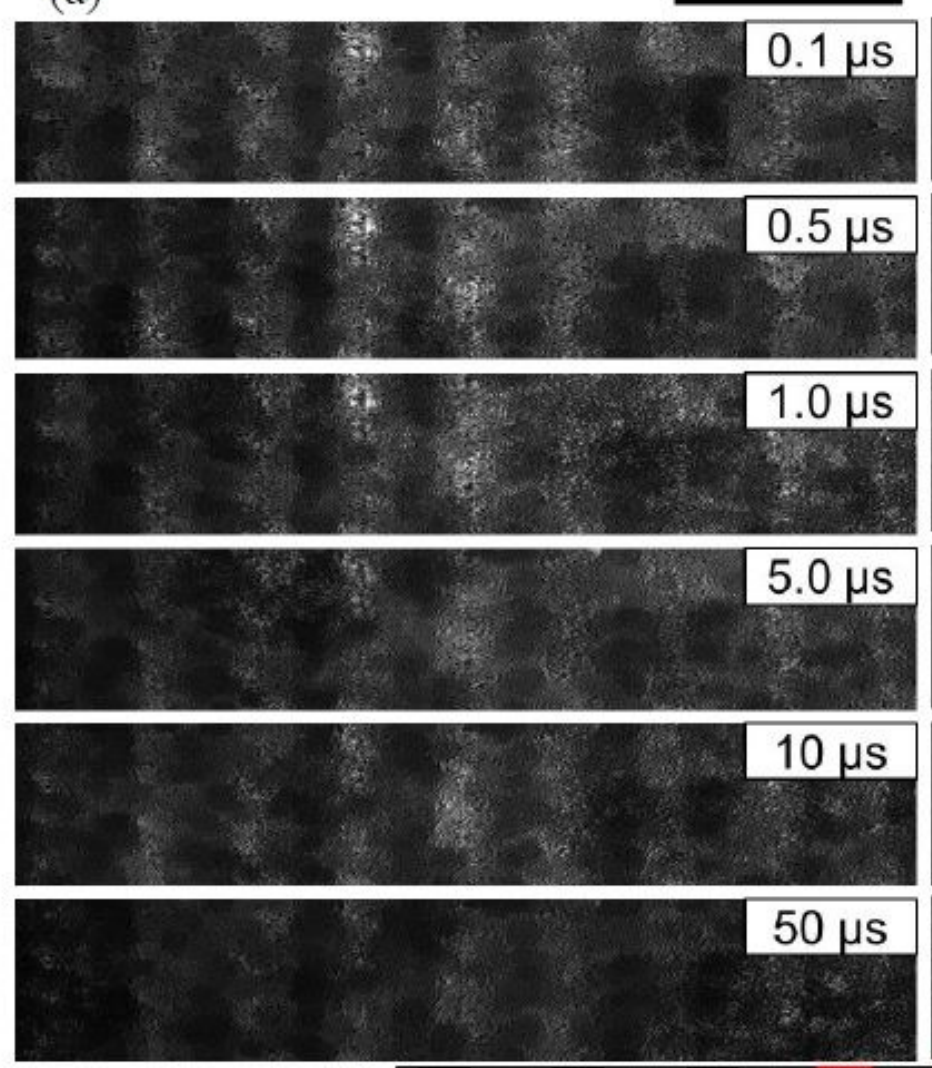

(c)
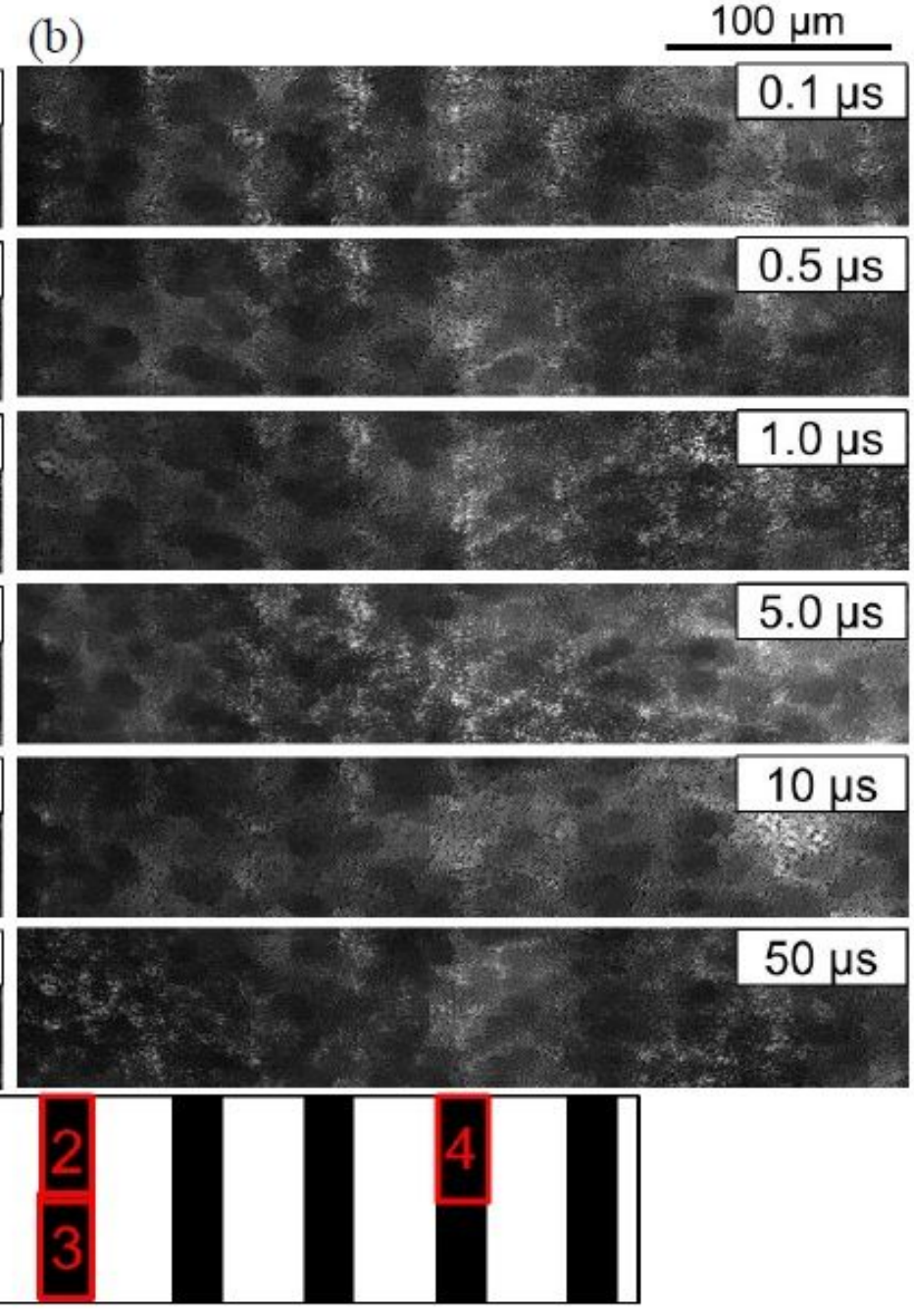

\section{Figure 1}

The image sequence of the refractive index change after photo-excitation by a UV pump light for SrTiO3:Rh/ITO/BiVO4:Mo (STOR/ITO/BVOM) Z-scheme water splitting sheet measured by the patternedillumination time-resolved phase microscope (PI-PM) in different solvents ((a) in ACN, (b) in water). (c) The light intensity pattern of the pump light and the selected regions for the cluster analysis is indicated in red squares. The result of the cluster analysis for area No.1 is shown in Fig.5-7, and the others are presented in Fig.S4-S8 in Supporting information (SI)). 
(a)

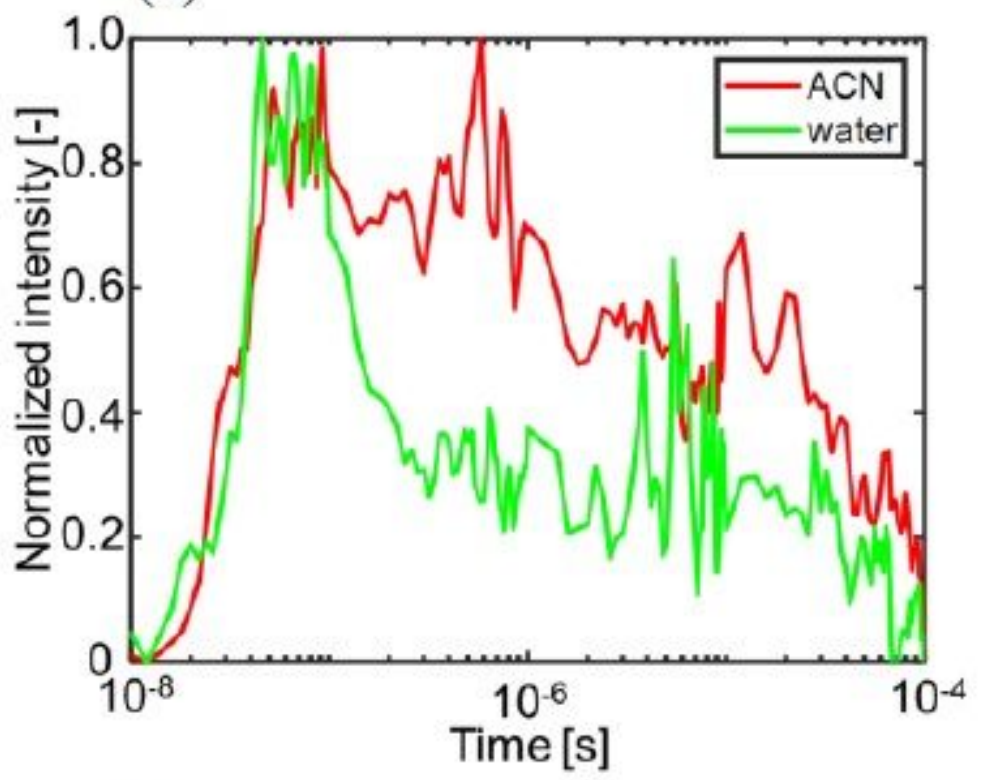

(b)

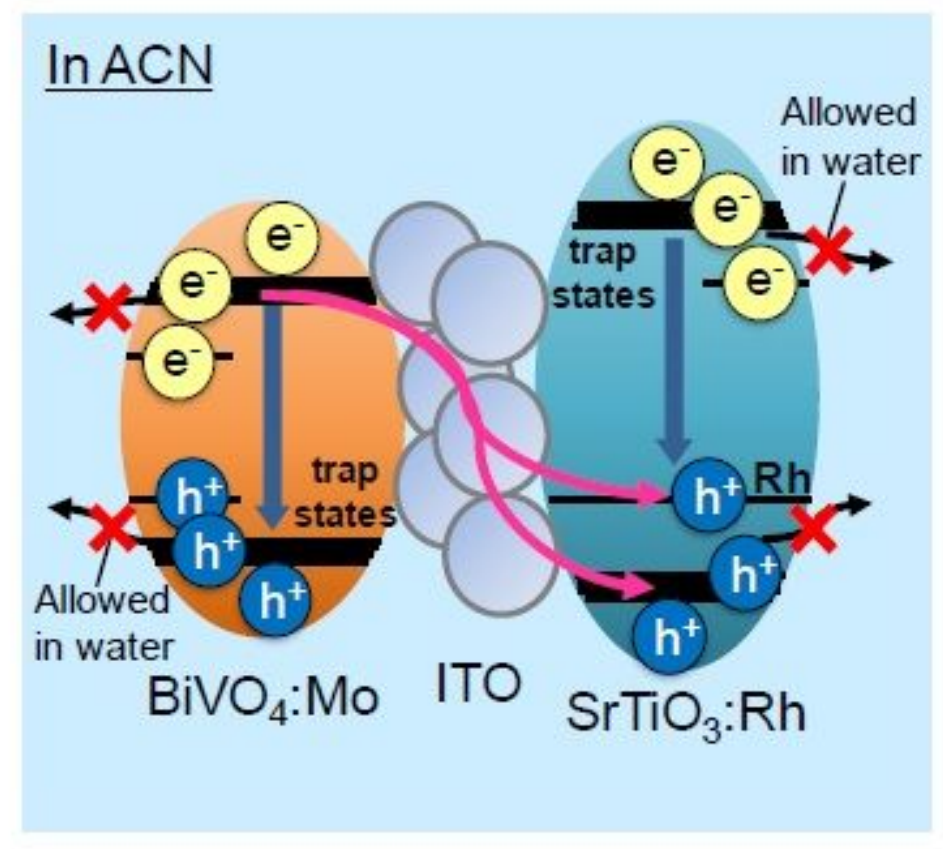

\section{Figure 2}

(a) The average refractive index change response of STOR/ITO/BVOM until $100 \mu$ s in acetonitrile (ACN) and water obtained from the PI-PM image sequence (Fig.1). The response was obtained from the signal amplitude at the light irradiated regions. (b) A schematic drawing of the charge transfer/decay processes for STOR/ITO/BVOM in ACN. Surface trapped carriers cannot be transferred to the solution side in ACN, where all the processes of charge dynamics are completed in the system. 

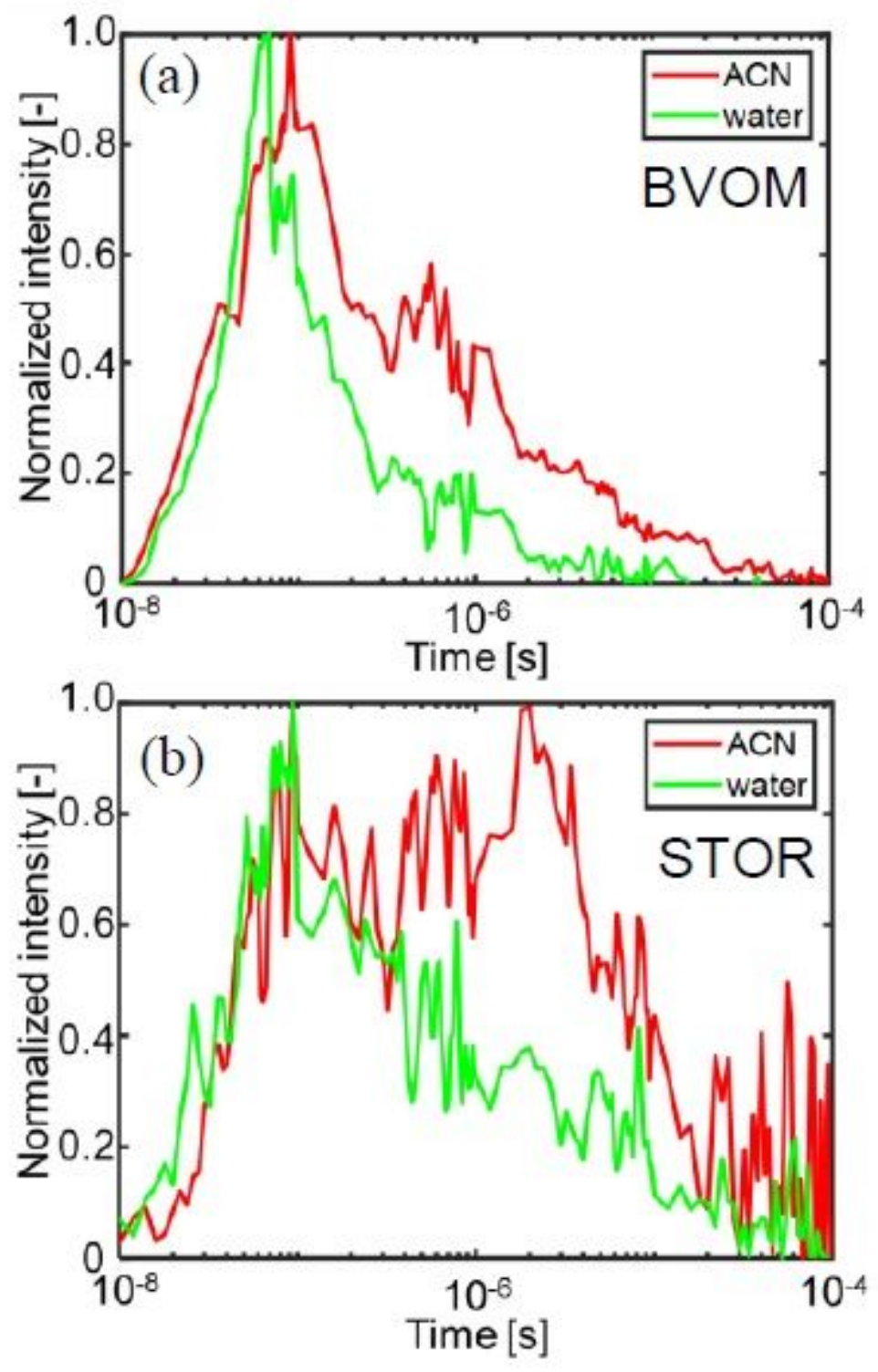

Figure 3

The average responses of the refractive index changes for (a) BVOM and (b) STOR until $100 \mu \mathrm{s}$ in ACN and water obtained by the PI-PM image sequences. 


\section{STOR/ITO/BVOM in $\mathrm{ACN}$}

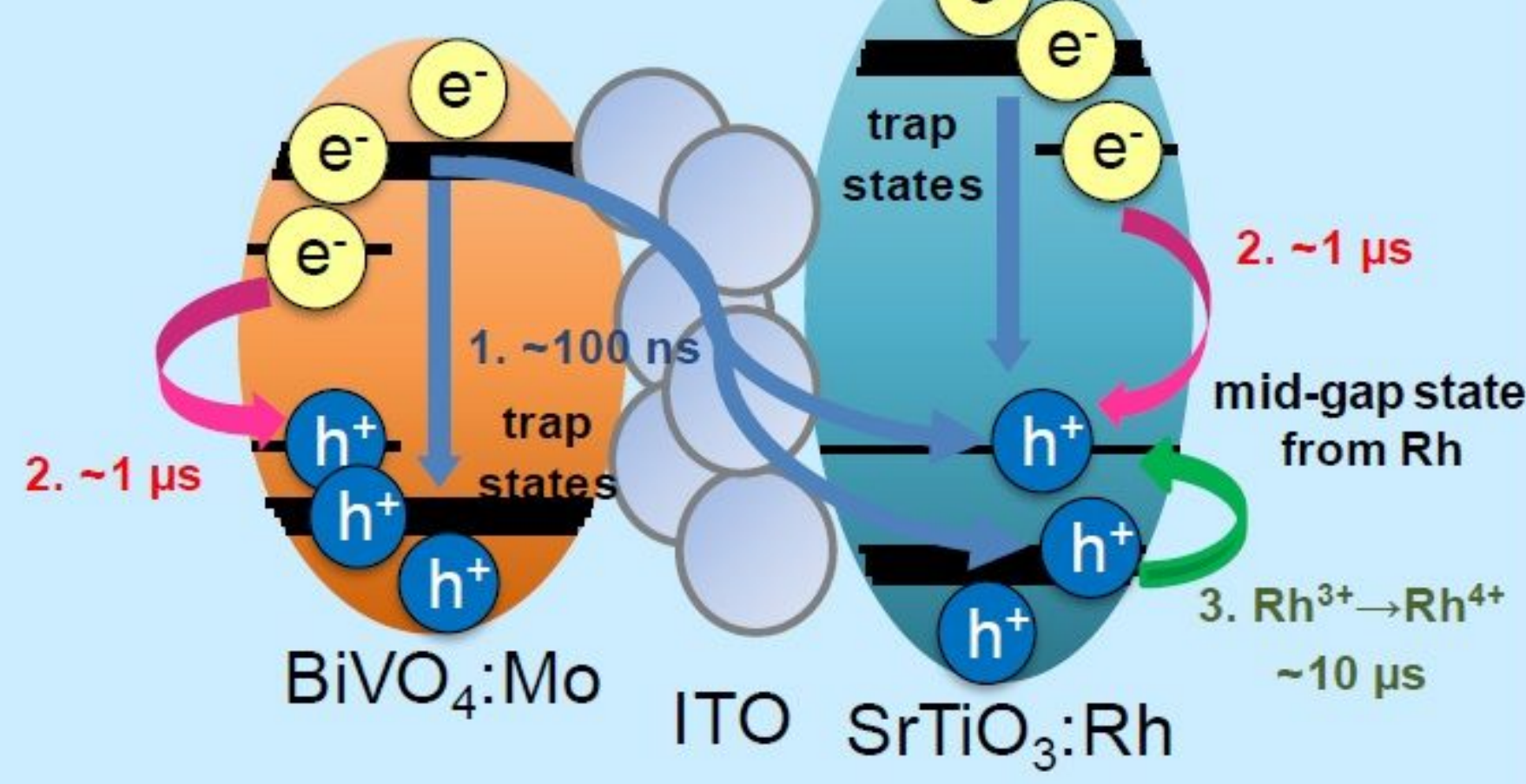

\section{Figure 4}

The schematic flow diagram of the whole charge dynamics in STOR/ITO/BVOM in ACN. Step 1 includes intrinsic recombination inside materials and charge transfer between two materials. Step 2 indicates the decay of surface trapped charge carriers in ACN, which are utilized for water splitting reactions with water outside. Step 3 includes the hole trapping to Rh3+/4+ state in STOR and the slower recombination. 


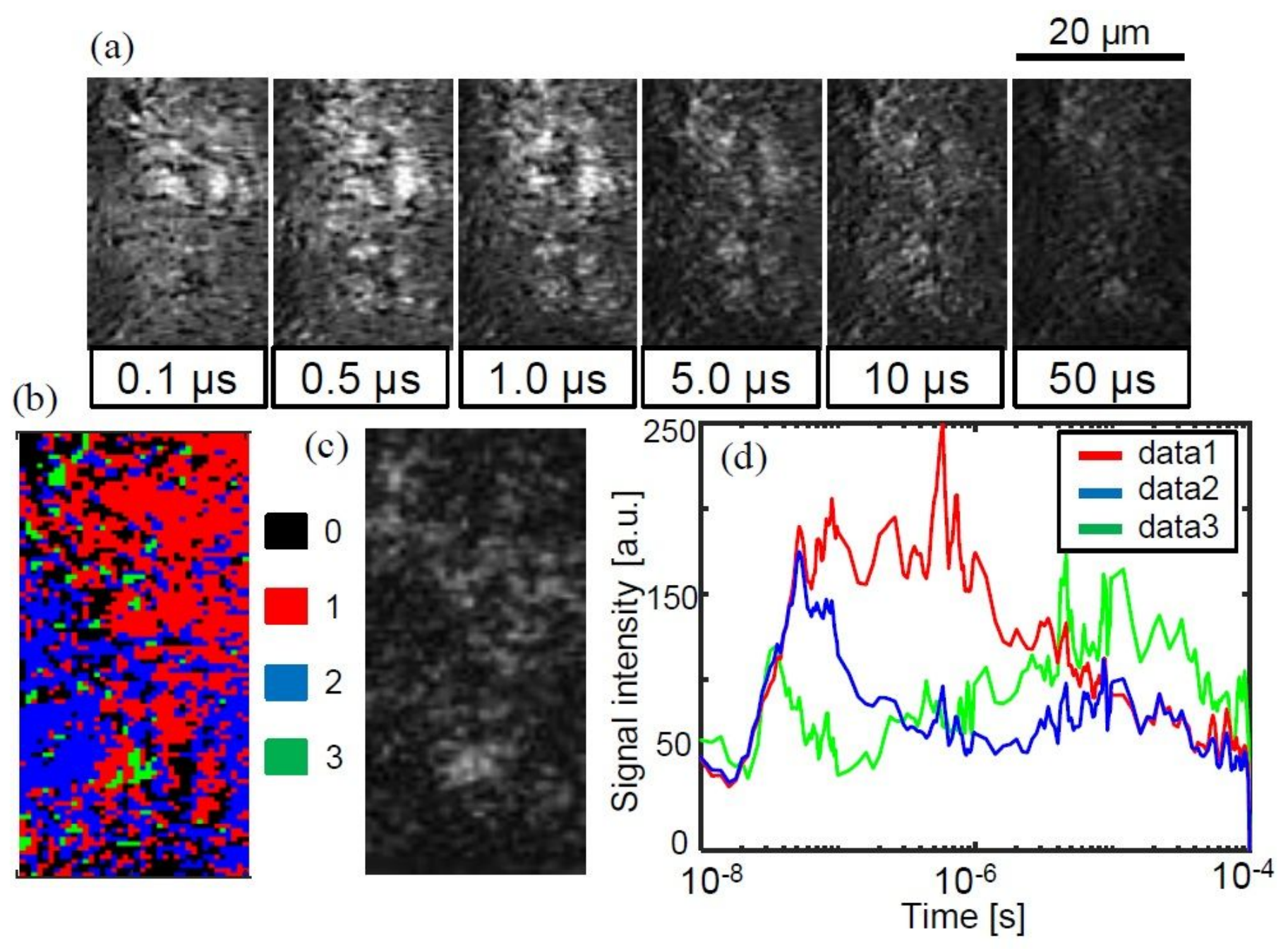

Figure 5

(a) An image sequence of the refractive index change for STOR/ITO/BVOM in ACN in a square region $(20 \times 50 \mu \mathrm{m})$ corresponding to No.1 in Fig.1(c) on the order from nanoseconds to microseconds. The scale bar corresponds to $20 \mu \mathrm{m}$. (b) The categorized mapping of the charge carrier responses of (a). An outlier positioned far from all categories were colored in black (\#0). (c) A microscopic image in the same area as (a). (d) The averaged responses for each category in (b) are shown. 


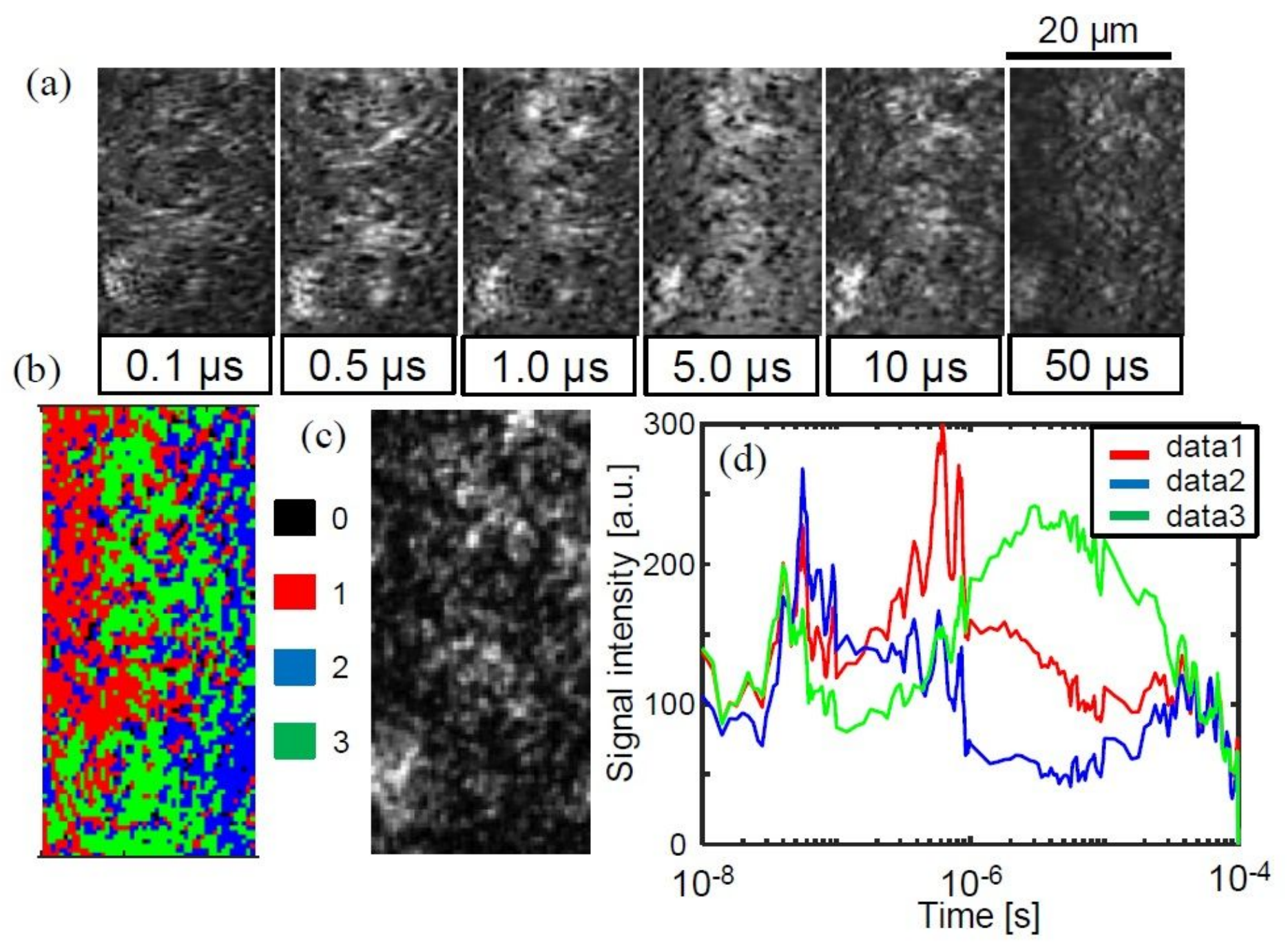

Figure 6

(a) An image sequence of the refractive index response for STOR/BVOM in ACN in a square region $(18 \times 50 \mu \mathrm{m})$ corresponding to No.1 in Fig.1(c) on the order from nanoseconds to microseconds. The scale bar corresponds to $20 \mu \mathrm{m}$. (b) The categorized mapping of the charge carrier responses of (a). An outlier positioned far from all categories were colored in black (\#0). (c) A microscopic image in the same area as (a). (d) The averaged responses for each category in (b) are shown. 

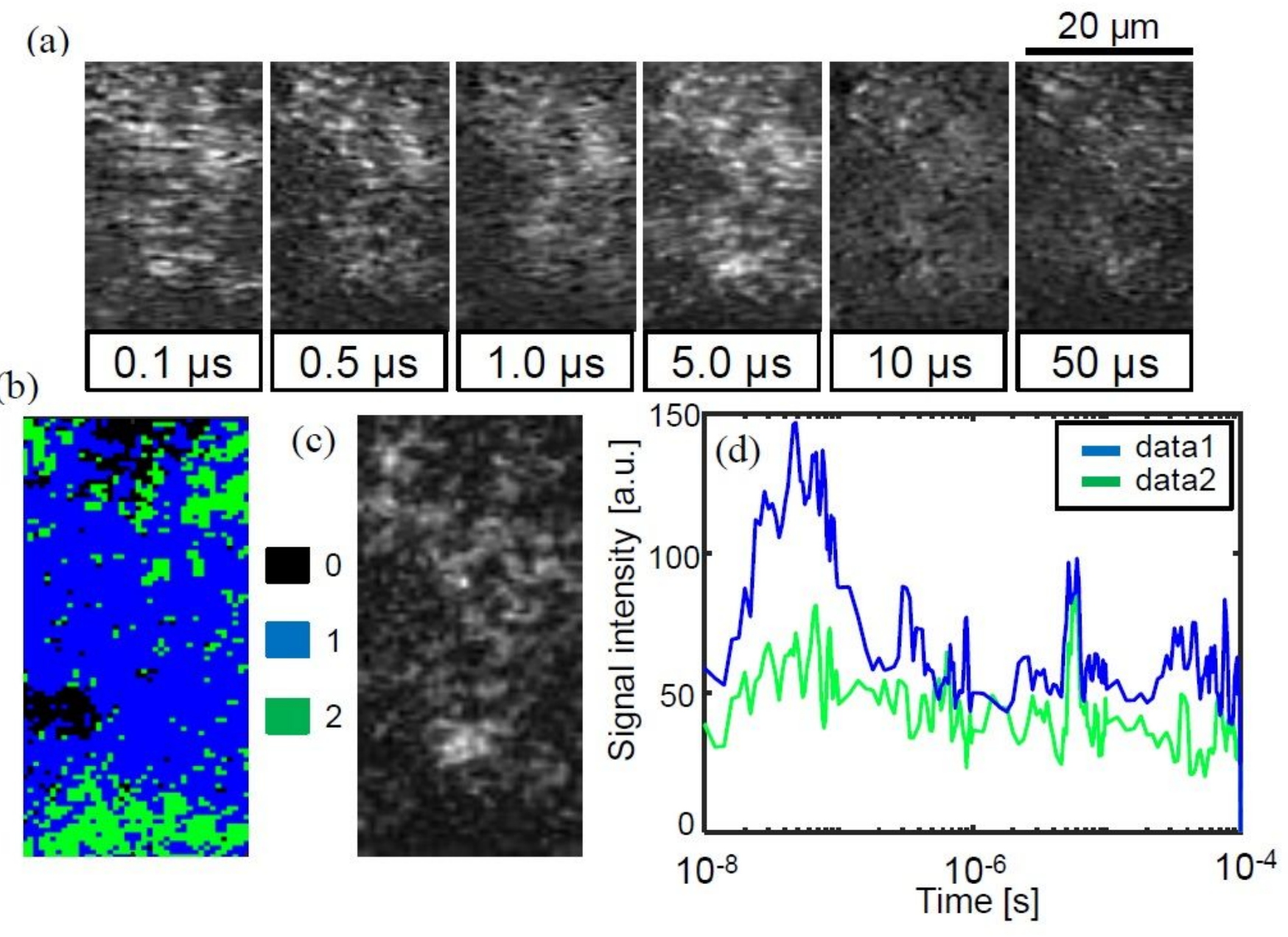

Figure 7

(a) An image sequence of the refractive index response for STOR/ITO/BVOM in water in a square region $(20 \times 50 \mu \mathrm{m})$ corresponding to No.1 on the order from nanoseconds to microseconds. The location of the sample was exactly the same as Fig.5(a). The scale bar corresponds to $20 \mu \mathrm{m}$. (b) The categorized mapping of the charge carrier responses of (a). An outlier positioned far from all categories was colored in black (\#0) (c) A microscopic image in the same area as (a). (d) The averaged responses for each category in (b) are shown.

\section{Supplementary Files}

This is a list of supplementary files associated with this preprint. Click to download.

- 20210225SupportinginformationZscheme.pdf 